\title{
Ustekinumab in the Treatment of Erythema Nodosum with Underlying Crohn's Disease: A Case Report
}

\author{
Antonio Sanín-Guana, BA¹, Mark G. Lebwohl, MD¹ \\ ${ }^{1}$ Department of Dermatology, Icahn School of Medicine at Mount Sinai, New York, NY
}

\section{ABSTRACT}

Erythema nodosum (EN) is a septal panniculitis characterized by raised, tender, red or purple lesions with a diameter of $1-5 \mathrm{~cm}$. It is commonly treated with corticosteroids and can be an extra-intestinal manifestation of Crohn's disease (CD). CD can be treated with ustekinumab, a monoclonal antibody that targets the p-40 subunit of the interleukin-12 (IL-12) and interleukin-23 (IL-23). A 78-year-old woman with erythema nodosum and Crohn's disease presented with multiple erythematous nodules, which had been recurring on both of her legs for decades. The patient had failed many traditional EN treatments and was prescribed off-label ustekinumab, resulting in resolution of EN. This finding suggests that ustekinumab may be a potential treatment for EN in patients who also have CD.

\section{INTRODUCTION}

Erythema nodosum (EN) is a septal panniculitis characterized by raised, tender, red or purple lesions with a diameter of 1-5 $\mathrm{cm}$. It is commonly treated with corticosteroids and can be an extraintestinal manifestation of Crohn's disease (CD). CD can be treated with ustekinumab, a monoclonal antibody that targets the $\mathrm{p}-40$ subunit of the interleukin-12 (IL-12) and interleukin-23 (IL-23).

\section{CASE REPORT}

A 78-year-old woman with a 25-year history of $C D$ which required colon resection and a 55-year history of poorly controlled EN presented with a chief complaint of painful bilateral lesions on her lower extremities, primarily on the right leg and dorsal feet. Similar lesions had been recurring for decades. The patient had a family history of ulcerative colitis, and her CD manifestations had included occasional perianal abscess, dysphagia, and acid reflux. Treatment for CD had included sulfasalazine and infliximab, and the patient failed treatments for EN, including oral potassium iodide, intralesional triamcinolone acetonide 10 $\mathrm{mg} / \mathrm{cc}$, and oral colchicine. On physical examination, she had 8 tender erythematous nodules on the anterior and posterior aspects of both lower legs. At the time of the visit, the patient had been receiving only prednisone $5 \mathrm{mg}$, with minimal improvement and could not be treated with NSAIDS because of her CD. Over the course of the next 11 months, she continued to develop intermittent nodules on both legs and feet. Although the patient was not actively experiencing any other $C D$ symptoms, the relationship of EN as an extra-intestinal manifestation of $C D$ suggested that the $C D$ was not well controlled. As such, a new

(c) 2022 THE AUTHORS. Published by the National Society for Cutaneous Medicine. 
treatment to target the underlying $C D$ could be initiated to attempt to resolve the EN, so the patient was prescribed off-label ustekinumab. Treatment began with a 180 $\mathrm{mg}$ infusion, and subsequent $90 \mathrm{mg}$ subcutaneous injections on average every 6 weeks. Improvement of the EN lesions was first seen at 16 months, and at 17 months there was full resolution of lesions, without development of new nodules. At 24 months, the patient had only scars on the lower legs.

\section{DISCUSSION}

Crohn's Disease (CD) is present in $0.2 \%$ of the adult population in the United States, presenting more commonly in women than in men. ${ }^{1}$ CD can manifest itself in any part of the gastrointestinal tract and is characterized by granulomatous transmural inflammation. CD symptoms generally include fever, fatigue, and weight loss. ${ }^{2}$ In addition to its typical intestinal symptoms, CD may also be associated with extraintestinal manifestations in $6-36 \%$ of patients. $^{2}$

Erythema nodosum (EN), the most widespread form of panniculitis (septal inflammation of subcutaneous fat tissue), ${ }^{8}$ is one such dermatological extraintestinal manifestation of $C D$ that is present in 4$15 \%$ of CD patients. ${ }^{3}$ It is three to five times more prevalent in female patients than in male patients and can occur amongst all age groups, although it generally is manifested in between the second and fourth decades of life. ${ }^{8} \mathrm{EN}$ is an inflammatory process that involves subcutaneous fat lobules, ${ }^{6}$ which cause raised, tender, red or purple lesions with a diameter of $1-5 \mathrm{~cm},{ }^{8}$ generally found on the anterior extensor surface of the lower extremities. ${ }^{4,5} \mathrm{EN}$ can be attributed to many different factors and may be idiopathic in up to $50 \%$ of cases, ${ }^{7}$ although in $1-4 \%$ of cases it may be caused by enteropathies, such as CD. ${ }^{8} \mathrm{~A}$ strong correlation exists between the presence of EN and intestinal disease activity. There are some reports that EN in patients with $C D$ is associated with the T-cell immune response to common antigens of intestinal and skin bacteria. The presence of ANCA and HLA-B27 antigen in patients with EN and IBD also argues in favor of the important role of genetic factors. ${ }^{8} \mathrm{EN}$ is usually treated with corticosteroids and intensification of medical therapy to control intestinal disease. ${ }^{5}$

Ustekinumab is a monoclonal antibody that targets the $\mathrm{p}-40$ subunit of the interleukin-12 (IL-12) and interleukin-23 (IL-23). ${ }^{9}$ It is approved by the FDA to treat moderate to severe plaque psoriasis and psoriatic arthritis, in addition to $\mathrm{CD}$ and UC. ${ }^{11}$ Inflammation of the gastrointestinal tract in CD patients has been associated with a Th1-type response; ustekinumab has been suggested to inhibit differentiation of Th1 cells in patients with CD. In addition, IL-12 and IL-23 have been implied in the differentiation of $\mathrm{T}$ follicular helper cells (TFH) in the pathogenesis of CD, and it has been suggested that ustekinumab therapy also affects TFH differentiation. ${ }^{10}$ In the literature, there is one other case reported of a 43-year-old female also affected with CD and $\mathrm{EN}$, who was treated with ustekinumab and experienced a total remission of her EN symptoms after 12 weeks of treatment. ${ }^{12}$

\section{CONCLUSION}

This is a case in which ustekinumab was effective in treating $\mathrm{EN}$ in a patient with concomitant $\mathrm{CD}$. This suggests that the inhibition of the IL-12 and IL-23 pathways in patients with CD may also be responsible for decreasing the inflammatory response 
observed in EN. As such, ustekinumab may present itself as a potential treatment for EN in patients who also have CD. However, more research needs to be done to examine this relationship and to evaluate the safety and efficacy of ustekinumab in treating any cases of EN, regardless of cause.

Conflict of Interest Disclosures: Mark Lebwohl is an employee of Mount Sinai and receives research funds from: Abbvie, Amgen, Arcutis, Avotres, Boehringer Ingelheim, Cara therapeutics, Dermavant Sciences, Eli Lilly, Incyte, Janssen Research \&amp; Development, LLC, Ortho Dermatologics, Regeneron, and UCB, Inc., and is a consultant for Aditum Bio, Almirall, AltruBio Inc., AnaptysBio, Arcutis, Inc., Arena Pharmaceuticals, Aristea Therapeutics, Arrive Technologies, Avotres Therapeutics, BiomX, Brickell Biotech, BoehringerIngelheim, Bristol-Myers Squibb, Cara Therapeutics, Castle Biosciences, Corevitas, Dermavant Sciences, Dr. Reddy's Laboratories, Evelo Biosciences, Evommune, Inc., Facilitatation of International Dermatology Education, Forte Biosciences, Foundation for Research and Education in Dermatology, Helsinn Therapeutics, Hexima Ltd., LEO Pharma, Meiji Seika Pharma, Mindera, Pfizer, Seanergy, and Verrica.

Funding: None

Corresponding Author:

Mark Lebwohl, MD

Icahn School of Medicine at Mount Sinai

5 E. 98th St., New York, NY 1029

Email: lebwohl@aol.com
4. Vavricka SR, Schoepfer A, Scharl M, Lakatos PL, Navarini A, Rogler G. Extraintestinal Manifestations of Inflammatory Bowel Disease. Inflamm Bowel Dis. 2015;21(8):1982-1992. doi:10.1097/MIB.0000000000000392

5. Fleisher M, Marsal J, Lee SD, et al. Effects of Vedolizumab Therapy on Extraintestinal Manifestations in Inflammatory Bowel Disease. Dig Dis Sci. 2018;63(4):825-833. doi:10.1007/s10620-018-4971-1

6. Weinstein M, Turner D, Avitzur Y. Erythema nodosum as a presentation of inflammatory bowel disease. CMAJ. 2005;173(2):145-146. doi:10.1503/cmaj.050201

7. Faulkes RE. Upper limb erythema nodosum: the first presentation of Crohn's disease. Clin Case Rep. 2014;2(5):183-185. doi:10.1002/ccr3.87

8. Chowaniec M, Starba A, Wiland P. Erythema nodosum - review of the literature. Reumatologia. 2016;54(2):79-82. doi:10.5114/reum.2016.60217

9. Feagan BG, Sandborn WJ, Gasink C, et al. Ustekinumab as Induction and Maintenance Therapy for Crohn's Disease. N Engl J Med. 2016;375(20):1946-1960. doi:10.1056/NEJMoa1602773

10. Globig AM, Sommer NP, Wild K, et al. Ustekinumab Inhibits T Follicular Helper Cell Differentiation in Patients With Crohn's Disease. Cell Mol Gastroenterol Hepatol. 2021;11(1):1-12. doi:10.1016/j.jcmgh.2020.07.005

11. Ustekinumab. Package insert. Janssen Pharmaceutical Companies; 2020.

12. Spagnuolo R, Dastoli S, Silvestri M, et al. Antiinterleukin 12/23 in the treatment of erythema nodosum and Crohn disease: A case report. Dermatol Ther. 2019;32(2):e12811. doi:10.1111/dth.12811

\section{References:}

1. Wilkins T, Jarvis K, Patel J. Diagnosis and management of Crohn's disease. Am Fam Physician. 2011;84(12):1365-1375.

2. Jumani L, Kataria D, Ahmed MU, Shah MAA, Raja K, Shaukat F. The Spectrum of Extra-intestinal Manifestation of Crohn's Disease. Cureus. 2020;12(2):e6928. Published 2020 Feb 10. doi:10.7759/cureus.6928

3. Annese V. A Review of Extraintestinal Manifestations and Complications of Inflammatory Bowel Disease. Saudi J Med Med Sci. 2019;7(2):66-73. doi:10.4103/sjmms.sjmms_81_18 